\title{
Fluid overload and length of mechanical ventilation in paediatric sepsis
}

Sepsis is a life-threatening clinical condition frequently faced by paediatricians in paediatric intensive care units (PICUs). One of the therapeutic approaches to sepsis is fluid resuscitation. However, critically ill children with sepsis have a higher risk of fluid accumulation due to increased capillary hydrostatic pressure and permeability. Fluid accumulation in the lung interstitium and alveoli may induce lung oedema and ventilation perfusion mismatch. In patients on mechanical ventilation, these conditions may result in prolonged ventilation. Previous studies have shown fluid overload could extend the length of mechanical ventilation (MV) and PICU stay. Fluid overload was also related to increased mortality rate in adult sepsis. The respiratory system (communityacquired pneumonia, $36.2 \%$ ), is the most common infection site found in paediatric sepsis globally. Other common systems involved are neurological problems and tropical infections (23.5\% and $17.4 \%$, respectively). Studies predicted that chronic septic children with preexisting chronic illness had higher risk of mortality than previously healthy children $(10.5 \%$ v. $8.1 \%$, respectively; $p<0.001)$. The aim of this study was to evaluate the correlation between fluid overload and the length of mechanical ventilation in children with sepsis admitted to the PICU. The primary outcome of this study was fluid overload percentage $(\% \mathrm{FO})$, defined as percentage of fluid balance (difference between fluid input and output) in the first 48 hours divided by body weight on admission. Secondary outcomes were PICU mortality and length of PICU stay. Eighty-eight percent of patients had fluid balance percentage of $<10 \%$ and $12.1 \%$ of the patients had $\geq 10 \%$ fluid overload. The median length of mechanical ventilation was 5 days, with the longest duration being 60 days. Fifty-two percent of the patients died in the PICU from septic shock. A previous study reported a significant correlation between fluid overload in the first 24 hours of care and mortality in 202 subjects (odds ratio (OR) 1.22; $95 \%$ confidence interval (CI) $1.12-1.33 ; p<0.001$ ). The mean duration of MV in the $\geq 10 \%$ FO group was longer than that of the $<10 \%$ FO group (56.72 v. 13.41 days) and there was a significant mean difference $(p=0.01)$. Age $<12$ months was the only factor significantly correlated with length of PICU stay ( $p=0.04)$. Mortality was significantly higher in the $\geq 10 \%$ FO group and affected by type of underlying disease.

In conclusion, fluid overload in paediatric sepsis significantly correlates with length of mechanical ventilation and PICU mortality, but not with length of PICU stay. More studies need to be conducted with a larger sample size taking into consideration the possibilities of difficulties in monitoring fluid balance in paediatric patients.

\section{P Mashiloane}

Paediatric Critical Care Fellow, University of KwaZulu-Natal and

Inkosi Albert Luthuli Central Hospital, Durban, South Africa

1. Paramitha W, Triasih R, Rusmawatiningtyas D. Fluid overload and length of mechanical ventilation in pediatric sepsis. Paediatr Indones 2019;59(4):211-216

\section{Mortality risk factors in children with severe influenza virus infection admitted to the paediatric intensive care unit}

Influenza virus infection is common in children. Approximately 90 million cases of influenza occur each year in children aged $<5$ years worldwide. About 870000 preschool children are hospitalised globally due to influenza virus infection each year. It may be self-limiting, but it can also lead to severe clinical outcomes or even death because of its complications. This study aimed to identify the mortality risk factors in children with severe influenza virus infections who were admitted to a aediatric intensive care unit. The most common period with the largest number of severe influenza infection cases was April to June. The complications of influenza infection included pneumonia (100\%), respiratory failure (96.1\%), acute respiratory distress syndrome (ARDS) (22.1\%), septic shock (15.6\%) and intracranial epidural abscesses (13.0\%). The influenza A to B ratio was 4.9:1.0. Bacterial co-infection was higher when compared with other reports. This was attributed to prolonged hospital stay and the use of fibro-bronchoscopy, and BAL cultures that had a better detection rate. Haemophilus influenzae and Streptococcus pneumoniae were the predominant bacterial coinfections and were associated with disease progression. The most common viral co-infection was adenovirus, contrary to other studies where respiratory syncytial virus was the commonest. Almost half of the patients needed prolonged ventilation for more than 7 days. The median time from onset of symptoms to initiation of oseltamivir therapy was 5 days. This was because of the difficulties faced with early detection of influenza A virus, as it has the same symptomology as other viral infections. Older age ( $>3$ years), oxygen saturation level $<90 \%$ at admission, ARDS, pneumorrhagia, intracranial epidural abscesses, and septic shock were associated with mortality risk (all $p<0.05)$. Hypoxia, deranged liver function test and elevated lactate dehydrogenase (LDH) were associated with mortality. The mortality from severe influenza infection was $16.9 \%$, which was comparable with other studies. Early recognition and management of severe infection may help to decrease PICU admission and mortality.

\section{P Mashiloane}

Paediatric Critical Care Fellow, University of KwaZulu-Natal and Inkosi Albert Luthuli Central Hospital, Durban, South Africa

1. Shi T, Nie Z, Huang L, et al. Mortality risk factors in children with severe influenza virus infection admitted to the pediatric intensive care unit. Med 2019;98(35):e16861. https://doi.org/10.1097/MD.0000000000016861 